\title{
Community Detection for Decoy Selection in Template-free Protein Structure Prediction
}

\author{
Liban Hassan \\ Dept of Computer Science \\ George Mason University \\ Nasrin Akhter \\ Dept of Computer Science \\ George Mason University
}

\author{
Zahra Rajabi \\ Dept of Computer Science \\ George Mason University \\ Amarda Shehu* \\ Dept of Computer Science \\ George Mason University
}

\begin{abstract}
Significant efforts are devoted to resolving biologically-active structures in wet and dry laboratories. In particular, due to hardware and algorithmic innovations, computational methods can now obtain thousands of structures that populate the structure space of a protein of interest. With such advances, attention turns to organizing computed structures to extract the underlying organization of the structure space in service of highlighting biologically-active structural states. In this paper we report on the promise of leveraging community detection methods, designed originally to detect communities in social networks, to organize protein structure spaces probed in silico. We report on a principled comparison of such methods along several metrics and on proteins of diverse folds and lengths. More importantly, we present a rigorous evaluation in the context of decoy selection in template-free protein structure prediction. The presented results make the case that network-based community detection methods warrant further investigation to advance analysis of protein structure spaces for automated selection of biologically-active structures.
\end{abstract}

\section{CCS CONCEPTS}

\section{- Applied computing $\rightarrow$ Molecular structural biology;}

\section{KEYWORDS}

protein structure space, nearest-neighbor graph, community detection, decoy selection

\section{ACM Reference Format:}

Liban Hassan, Zahra Rajabi, Nasrin Akhter, and Amarda Shehu. 2018. Community Detection for Decoy Selection in Template-free Protein Structure Prediction. In ACM-BCB'18: 9th ACM International Conference on Bioinformatics, Computational Biology and Health Informatics, August 29-September 1, 2018, Washington, DC, USA. ACM, New York, NY, USA, 7 pages. https: //doi.org/10.1145/3233547.3233703

\footnotetext{
${ }^{*}$ Corresponding Author: amarda@gmu.edu

Permission to make digital or hard copies of all or part of this work for personal or classroom use is granted without fee provided that copies are not made or distributed for profit or commercial advantage and that copies bear this notice and the full citation on the first page. Copyrights for components of this work owned by others than ACM must be honored. Abstracting with credit is permitted. To copy otherwise, or republish, to post on servers or to redistribute to lists, requires prior specific permission and/or a fee. Request permissions from permissions@acm.org.

ACM-BCB'18, August 29-September 1, 2018, Washington, DC, USA

(C) 2018 Association for Computing Machinery.

ACM ISBN 978-1-4503-5794-4/18/08 . \$ \$15.00

https://doi.org/10.1145/3233547.3233703
}

\section{INTRODUCTION}

With tertiary structure long recognized as central to the biological activities of a protein in the cell [5], significant efforts in molecular biology are devoted to probing protein structure spaces. In principle, dry-laboratory efforts can provide a detailed view of the structure space of a protein of interest. The most visible work in this direction is under template-free protein structure prediction [8, 16, 26, 32], which, provided a protein amino-acid sequence, computes tertiary structures that are local minima of some selected energy function. Other methods operationalizing data-driven modeling of structures and structural dynamics provide information on a protein's structure space and its traversal [7, 20,22,30].

Software and hardware advances have resulted in an explosion of structure data; it is now possible to generate dozens (and even hundreds) of thousands of tertiary protein structures for a given protein sequence in a matter of a few days, leveraging embarrassing parallelism in supercomputer architectures [21]. Currently, there is a need for methods that can uncover the organization of the protein structure space probed in silico. Such organization can reveal, for instance, the grouping of structures in different thermodynamicallystable and semi-table states. A narrow version of this problem is known as decoy selection in template-free protein structure prediction; the computed structures are referred to as decoys, and the goal is to discover the biologically-native/native structure among the decoys computed. Even in this narrow context, which only considers one stable state, the problem remains open [15].

In this paper we seek to elucidate the organization of the protein structure space probed by a computational method. We focus here on tertiary, all-atom structure data obtained via the Rosetta $a b$ initio protocol as a state-of-the-art and representative template-free protein structure prediction method. Specifically, we leverage a graph-based organization of tertiary structures upon which we can then identify communities by leveraging and adopting ideas from community detection in complex networks (such as social networks). We evaluate state-of-the-art, representative community detection methods for their ability to identify communities along an extensive list of community-accepted metrics. Moreover, we evaluate here the promise of leveraging the identified communities for decoy selection in template-free protein structure prediction.

\subsection{Related Work}

We summarize here research on decoy selection, which is evaluated in the Critical Assessment of protein Structure Prediction (CASP) 
series of community wide experiments [24]. Current decoy selection methods can be grouped into single-model, bag-of-models, quasisingle methods, and machine learning (ML) methods.

Single-model methods assess the quality of one tertiary structure at a time $[17,31]$ via a physics- or knowledge-based energy/scoring function. Though statistical functions are better able to distinguish native from non-natives structures [11], all single-model methods can be utilized for decoy selection by setting a score threshold; this is unreliable, as the threshold misses native structures or allows the inclusion of too many non-native ones [2]. In response, it is popular to ignore energy altogether and cluster decoys by structural similarity [18, 34], offering the top highest-populated clusters as prediction. Cluster-based decoy selection implements the bag-of-models approach, operating under the premise that decoys are randomly distributed around the "true answer," which a consensus-seeking method ought to reveal. However, this is not a valid assumption. Template-free methods employ heuristics that bias decoy generation away from uniform sampling of the structure space. In addition, employed energy/scoring functions that guide the sampling contain inherent biases that often invalidate entire regions of the space. Cluster-based methods are shown to fail to pick up exceptionally-good decoys and to be weak when applied to hard targets where most decoys are very different from the known native structure(s), highly dissimilar, and sparsely sampled [24].

Quasi single-model methods adopt strategies from single-model and bag-of-models methods, selecting first some high-quality structures to which then to compare the rest of the decoys [14]. These methods improve decoy selection over single-model methods and consensus-seeking methods [13, 27]. In addition, a number of machine learning (ML) models such as Support Vector Machines (SVM) [6], Neural Network [25], and Random Forest [19] have proven to be competitive in decoy selection. Ensemble learning techniques have recently shown promise [23] over SVM learning, with or without statistical features. Though in their infancy, ML methods are showing promise and warrant further evaluation.

\section{METHODS}

In summary, we propose the following approach to detect organization in a computationally-probed protein structure space: computed structures of a protein of interest are embedded in a graph, which is then subjected to community detection algorithms that identify cohesive groupings of structures, or communities. To evaluate the utility of such an organization, we then demonstrate how various techniques leverage properties of identified communities to automatically select a subset of communities more likely to contain functionally-relevant structures. We demonstrate the promise of such an approach in the context of decoy selection. Though the approach is general, below we refer to structures as decoys. A description of the main steps of the proposed approach follows.

\subsection{A Graph-based Embedding of Decoys}

One can encode the proximity of decoys in the structure space via a nearest-neighbor graph (nngraph). Consider an $\Omega$ set of decoys (more generally, these can be uncomplexed or complexed molecular structures). $\Omega$ can be embedded in a nearest-neighbor graph (nngraph) $G=(V, E)$, as follows. The vertex set $V$ is populated with the decoys, and the edge set $E$ is populated by inferring a local neighborhood structure over each decoy. The distance between two decoys is measured via root-mean-squared-deviation (RMSD) after each of the decoys are superimposed over some reference decoy (arbitrarily, chosen to be the first in the generated decoy ensemble); the superimposition minimizes differences due to rigid-body motions. Superimposing all decoys to a reference a priori to the pairwise RMSD computation rather than seeking an optimal alignment for each pair of decoys under comparison saves computational time (from linear to quadratic). Using RMSD to compute the distance between two decoys, each vertex $u \in V$ is connected to vertices $v \in V$ if $d(u, v) \leq \epsilon$, where $\epsilon$ is a user-defined parameter. We utilize proximity query data structures (such as a kd-tree) to efficiently extract the nearest neighbors of a vertex.

A small $\epsilon$ may result in a disconnected graph, which is often the result of a sparse, non-uniform sampling of the structure space. This can be remedied by initializing $\epsilon$ to an initial value of $\epsilon_{0}$ and then increasing it by $\delta \epsilon$ over a maximum of $n_{\epsilon}$ iterations, while controlling the density of the resulting nngraph via a maximum number, $k$, of nearest neighbors per vertex. In this way, only vertices that have less than $k$ neighbors gain neighbors after each iteration.

The resulting nngraph is now investigated for its organization via community detection methods. We consider a thorough list of 7 representative, state-the-art methods, described below.

\subsection{Community Detection Methods}

Edge betweenness (Girvan-Newman): The method operationalizes the observation that edges connecting communities are expected to have high edge betweenness, which generalizes Freeman's betweenness centrality [12]. The underlying community structure is identified after removing edges with high edge betweenness. The calculation of edge betweenness for an edge is $O(|E| \cdot|V|)$, yielding an overall time complexity of $O\left(|E|^{2} \cdot \mid V\right)$.

Leading Eigenvector (LE): This method aims to maximize modularity over possible partitions of a network by utilizing the eigenspectrum of the modularity matrix [10]. At each step, the method finds a leading eigenvector which subdivides the graph into two clusters provided that the modularity improvement is maximized. The method stops if the contribution of the modularity change in the subdivided network becomes negatives. The overall time complexity for each partitioning is $O(|V|(|E|+|V|))$.

Walktrap (WT): This method utilizes random walks to capture structural similarities between vertices (or sets of vertices). The idea is that vertices within the same community are expected to have shorter-distance random walks. The method implements an agglomerative approach that starts from $|V|$ communities (reduced to a single vertex), hierarchically combining two adjacent communities at a time. The method runs in $O\left(|E||V|^{2}\right)$.

Label Propagation (LP): In this method, each vertex in the network is assumed to join the same community as the majority of its neighbors [28]. The method uses the network structure without optimization of a predefined objective function or any prior knowledge about the communities. First, each vertex is given a unique label (starting with $|V|$ communities). The labels are iteratively propagated through the network, reaching a consensus on a unique 
label. The stop criterion is reached once each vertex has the same label as the majority of its neighbors. The method runs in $O(|E|)$.

Louvain (Lo): This method is based on modularity optimization [4] and has two stages: In the first stage, the method starts with an initial partition in which each vertex is assigned a different community. Modularity gain is calculated when a vertex is assigned to a different community. Ordering of the vertices is shown not to influence modularity but to affect computation time. In the second stage, a new, weighted network is built, whose vertices are now the communities. This process continues iteratively until there are no changes, and a maximum of modularity is attained.

InfoMap (IM): In this method, communities are identified using random walks to analyze the information flow [29]. The network is encoded into modules that maximize the amount of information in the original network. A signal is sent to a decoder through a channel with limited capacity. The decoder tries to decode the message and to construct possible candidates for the original network. The smaller the number of candidates, the more information about the original network has been transferred. This method runs in $O(|E|)$

Greedy Modularity Maximization (GMM): This method uses a greedy optimization approach, assuming that high values of modularity correspond to good partitions of a network into communities [9]. Initially, each vertex is its own community. Vertices are then repeatedly joined together into two communities, whose modularity produces the largest increase. This process is represented by a tree structure (a dendrogram), whose leaves are the vertices of the original network, and whose internal vertices correspond to the joins. This method runs in $O(|E| d \log |V|)$, where $d$ is the depth of the dendrogram describing the network's community structure.

\subsection{Metrics for Evaluating Community Detection Methods}

We consider the following comprehensive list of 15 recommended metrics to evaluate community detection methods [33]. These metrics are scoring functions that mathematically formalize the community-wise connectivity structure of a given set of vertices and characterize high-scored sets as communities. For the purpose of summarizing these metrics below, let us suppose we have a an undirected graph $G(V, E)$, which has $n=|V|$ vertices and $m=|E|$ edges, and a community is a set $S$ of $n_{S}$ vertices with $m_{S}$ edges.

Fraction Over Median Degree (FOMD): If $d(u)$ represents the degree of each vertex $u \in S$ and $d_{m}$ the median value over degrees $d(u)$, FOMD is the fraction of vertices in $S$ that have internal degree higher than $d_{m}$, thus defined as: $f(S)=\frac{\left|\left\{u: u \in S,|\{(u, v): v \in S\}|>d_{m}\right\}\right|}{n_{S}}$ FOMD scores higher the denser, more cohesive communities.

Max ODF (Out Degree Fraction): This measures the maximum fraction of edges of a vertex in $S$ that point outside $S$, as: $f(S)=$ $\max _{u \in S} \frac{|\{(u, v) \in E: v \notin S\}|}{d(u)}$. The metric characterizes a community as a set of vertices that link to more vertices inside the community than to vertices. Lower scores correspond to better communities.

Triangle(Triad) Participation Ratio: This is the fraction of vertices that belong to a triangle, where $T_{c}$ is the number of vertices that form a triangle in $S$, and is defined as:

$f(S)=\frac{|\{u: u \in S,\{(v, w): v, w \in S,(u, v) \in E,(u, w) \in E,(v, w) \in E\} \neq \emptyset\}|}{n_{S}}$. Higher scores correspond to better community clustering.
Internal Edge Density: This metric is based on the internal connectivity of a cluster. Let us say $m_{S \max }=n_{S}\left(n_{S}-1\right) / 2$ represents the maximal number of possible edges for the set $S$. The edge density is the fraction of these edges which are actually present in $S$, defined as: $f(S)=\frac{m_{S}}{n_{S}\left(n_{S}-1\right) / 2}$. A higher score corresponds to more connectivity among vertices of a community.

Average Internal Degree: This measures the average internal degree of members of $S$ and is defined as: $f(S)=\frac{2 m_{S}}{n_{S}}$. Higher scores correspond to denser communities.

Cut Ratio: This measures the fraction of existing edges (out of all possible edges) leaving the community, and is defined as: $f(S)=\frac{C_{S}}{n_{S}\left(n-n_{S}\right)}$, where $C_{S}$ represents edges pointing out of $S$. Lower scores correspond to better communities.

Expansion: This measures the number of edges per vertex that point outside of a community $S$, and is defined as: $f(S)=\frac{C_{S}}{n_{S}}$. Lower scores represents higher community-like sets of vertices.

Edges Inside: This is the number of edges in $S$, and is defined as $f(S)=m_{S}$. Higher scores correspond to better communities.

Conductance: This measures the fraction of the total edge volume that points outside a community, and is defined as: $f(S)=$ $\frac{C_{S}}{\left(2 m_{S}+C_{S}\right)}$, Where $C_{S}$ is the number of edges pointing outside. Lower values correspond to better separated communities.

Normalized Cut: This metric is defined as: $f(S)=\frac{C_{S}}{\left(2 m_{S}+C_{S}\right)}+$ $\frac{C_{S}}{2\left(m-m_{S}\right)+C_{S}}$. It simultaneously satisfies both maximizing dissimilarity among communities and minimizing total similarity, avoiding the unnatural bias for partitioning out small sets. Lower values balance between the goal of finding clumps and splits.

Coverage: This is the ratio of the number of intra-community edges to $|E(G)|=m$, and is defined as: $f(S)=\frac{\omega(C)}{\omega(G)}$, Where $\omega(C)=\sum_{i=1}^{k} \omega\left(E\left(v_{x}, v_{y}\right)\right) ; v_{x}, v_{y} \in C_{i}$. Higher values mean that there are more edges inside the communities than edges linking different communities; ideally, communities are disconnected from one another, yielding a maximum coverage of 1 .

Average ODF This is the average fraction of edges of vertices in S that point out of S, and is defined as: $f(S)=\frac{1}{n_{S}} \sum_{u \in S} \frac{|\{(u, v) \in E: v \notin S\}|}{d(u)}$. Lower values correspond to better communities.

Modularity: This measures the difference of the number of edges within $S$ and the expected number of such edges in a random graph with identical degree sequence, and is defined as: $f(S)=$ $\frac{1}{4}\left(m_{S}-E\left(m_{S}\right)\right)$. Higher modularity represents denser connections in a community than would be expected at random.

Flake ODF: This measures the ratio of the number of vertices which have fewer edges pointing inside than to the outside of a community, and is defined as: $f(S)=\frac{|\{u: u \in S,|\{(u, v) \in E: v \in S\}|<d(u) / 2\}|}{n_{S}}$. Higher values correspond to better communities.

Separability: This is a popular metric [33] that measures the ratio between edges in $S$ and edges pointing outside $S$, and is defined as: $f(S)=\frac{m_{S}}{C_{S}}$. Higher separability corresponds to better communities.

\subsection{Community Selection for Decoy Selection}

Different measurements can be associated with the communities identified by any of the above methods. Two such measurements are size and energy. Size refers to the number of decoys/vertices 
in an identified community. The energy of a community can be defined in several ways; we consider two options. The energy of a community can be the minimum energy over all decoys in it, or it can be the average over the energies of the decoys in it.

Given size and energy, the communities can be ranked. For instance, considering only size, the communities can be ranked in a descending sorted order, and an automatic "community selection" strategy can extract the top $c$ communities and offer them as "prediction" for where native and near-native structures reside. We refer to this selection strategy as Sel-S. Another ranking strategy can additionally consider energy; considering energy alone has long been proven ineffective, as summarized in Section 1. So, instead, in Sel-S+E, we consider the top $l>c$ largest communities (in the sorted order), and then re-sort these $l$ communities from lowest to highest energy, selecting the top $c$ of them for prediction.

We consider two more selection strategies that are based on multi-objective optimization and leverage the concept of Pareto optimality. We note the unclear interaction between the size and energy of a community. So, we employ the concept of dominance to assist with selection based on possibly conflicting criteria.

Specifically, suppose that we want to select optimally considering various conflicting criteria/objectives. In this scenario, Paretooptimal solutions are sought, as a single solution minimizing all conflicting objectives simultaneously is typically non-existent; a Pareto-optimal solution cannot be improved in one objective without sacrificing the quality of at least one other objective. In other words, a solution $S_{1}$ Pareto-dominates another solution $S_{2}$ if the following two conditions are satisfied: (1) For all optimization objectives $i$, $\operatorname{score}_{i}\left(S_{1}\right) \geq \operatorname{score}_{i}\left(S_{2}\right)$; (2) For at least one optimization objective $i$, score ${ }_{i}\left(S_{1}\right)>$ score $_{i}\left(S_{2}\right)$.

One can now associate two additional quantities, Pareto Rank $(\mathrm{PR})$ and Pareto Count $(\mathrm{PC})$ with each community $C$. $\mathrm{PR}(C)$ is the number of communities that dominate $C . \mathrm{PC}(C)$ is the number of communities that $C$ dominates. So, two additional, Pareto-based selection strategies are now proposed. In Sel-PR, the communities are sorted by low to high PR values, and the top $c$ communities in this sorted order are selected and analyzed. In Sel-PR+PC, PC is additionally considered. Communities with the same $\mathrm{PR}$ value are sorted from high to low PCs, and the top $c$ communities in this resulting sorted order are selected and analyzed.

\subsection{Evaluating Selected Communities}

The quality of a set of decoys offered as "prediction" can be evaluated based on the number of near-native decoys contained in them: (1) $n$ - the percentage of near-native decoys in the selected set relative to the overall number of near-native decoys in the entire decoy ensemble, and (2) $p$ - the percentage of near-native decoys in the selected set over the number of decoys in the set. These metrics are inspired by machine learning performance metrics. Purity penalizes a large sets that, due to its size, may contain a large number of true positives (near-native decoys) but also a high number of false positives (non-native decoys). The reason for penalizing the number of false positives is by envisioning that a community selection strategy (which offers one or more communities as a predicted set) may be utilized for further discovery. If a set of decoys is presented to contain the true answer, but the majority of decoys in it are false positives, then the ratio of noise to signal is too high to be useful. On the contrary, a set of decoys with more near-native decoys in them is a better "prediction," as the likelihood of selecting a near-native decoys by drawing uniformly at random is higher when the number of false positives, non-native decoys, is low. We emphasize that if $c>1$ communities are offered as "prediction," the decoys from these communities are combined (which we note via $C_{1-m}$ ), and the resulting set is evaluated via the $n$ and $p$ metrics.

To determine whether a decoy is considered near-native or nonnative, an RMSD threshold is utilized. The selected threshold needs to allow populating the positive data set (non-zero number of nearnative decoys), which can then be used to evaluate a decoy selection strategy. A threshold dist_thresh is used and is set on a pertarget basis, as there are protein targets on which the quality of generated decoys suffers greatly from either the size and/or fold of the protein under investigation. In our drawing of a list of proteins on which to evaluate the premise of community detection for decoy selection, we consider proteins that are easy, medium, and hard in their difficulty for Rosetta structure prediction. For instance, we include in our evaluation cases where Rosetta does not get close to $3 \AA$ of the known native structure. Specifically, we consider the following thresholds: If the lowest IRMSD from a given native structure (over all decoys), to which we refer as min_dist, is $\leq$ 0.7 (these are considered easy cases), dist_thresh is set to $2 \AA$. Otherwise, dist_thresh is set to the minimum value that results in a non-zero number of near-native decoys populating the largestsize cluster obtained via leader clustering; the latter is used as a baseline in our comparison of community selection to cluster-based selection. For medium-difficulty proteins $(0.7 \AA<$ min_dist $<2 \AA)$, dist_thresh varies between $2-4.5 \AA$. We set dist_thresh to $6 \AA$ if min_dist $\geq 2 \AA$ (these are the hard cases). This ensures a non-zero number of near-native decoys to evaluate decoy selection strategies. A detailed analysis of the impact of this threshold on cluster-based decoy selection is related in our recent work [1].

\subsection{Implementation Details}

All in-house codes are implemented in Python. In the nngraph construction, $\delta \epsilon=0.2 \AA, k=20, n_{\epsilon}=5$, and $\epsilon_{0}$ is set so as not to exceed $900 \mathrm{~K}$ edges, varying in $0.5-2.2 \AA$ for most of the test cases, with one particularly challenging case for Rosetta set to $6.0 \AA$. The nngraph construction takes between 26 minutes to 4.25 hours on one CPU. We select the kd-tree over the vp-tree for fast extraction of nearest neighbors, based on analysis of the time demands as a function of dimensionality (data not shown). The community detection methods take between 7 minutes and 35 hours using 8-cores and 1GB memory per core. In community selection, $1 \leq c \leq 3$.

\section{RESULTS}

We conduct our evaluation on 9 proteins of different folds and lengths (number of amino acids), listed in Table 1. The decoy ensemble of each protein is generated from its amino-acid sequence, using the Rosetta ab-initio protocol [16]. The Mason Argo supercomputing cluster is utilized to execute the protocol around 50, 000 times in an embarrassingly parallel fashion to obtain ensembles of around 50,000 decoys per protein; the actual sizes are shown in 
Column 5 in Table 1. Column 3 shows the Protein Data Bank [3] identifier for a known, crystallographic structure for each protein.

The test cases listed in Table 1 are divided into three categories (easy, medium, and hard). This categorization is made evident by findings reported later, but it also emerges from analysis in terms of the lowest (1)RMSD over all decoys from the corresponding native structure. The lowest IRMSD over the decoys for each protein is listed in Column 6 in Table 1 (referred to as min_dist).

Table 1: Column 2 shows the PDB ID of a known native structure for each test case. Columns 3 and 4 show the fold ( ${ }^{*}$ indicates native structures with a predominant $\beta$ fold and a short helix) and the length (number of amino acids), respectively. Column 5 shows the size of the decoy set $\Omega$ generated via Rosetta, and column 6 shows the lowest IRMSD from the known native structure over the decoy ensemble.

\begin{tabular}{|c|c|c|c|c|c|c|}
\hline & & $\begin{array}{l}\text { PDB } \\
\text { ID }\end{array}$ & Fold & $\begin{array}{l}\text { Length } \\
\text { (\# aas) }\end{array}$ & $|\Omega|$ & $\begin{array}{l}\text { min_dist } \\
\text { (A) }\end{array}$ \\
\hline \multirow{3}{*}{ Easy } & 1. & $1 \mathrm{dtdb}$ & $\alpha+\beta$ & 61 & 57,839 & 0.51 \\
\hline & 2. & 1 tig & $\alpha+\beta$ & 88 & 52,099 & 0.60 \\
\hline & 3. & $1 \mathrm{dtja}$ & $\alpha+\beta$ & 74 & 53,526 & 0.68 \\
\hline \multirow{3}{*}{ Medium } & 4. & 1hz6a & $\alpha+\beta$ & 64 & 57,474 & 0.72 \\
\hline & & $1 \mathrm{c} 8 \mathrm{ca}$ & $\beta^{*}$ & 64 & 53,322 & 1.08 \\
\hline & 6. & 1sap & $\beta$ & 66 & 51,209 & 1.75 \\
\hline \multirow{3}{*}{ Hard } & & 2ezk & $\alpha$ & 93 & 50,192 & 2.56 \\
\hline & & 1 aoy & $\alpha$ & 78 & 52,218 & 3.26 \\
\hline & 9. & 1isua & coil & 62 & 60,360 & 5.53 \\
\hline
\end{tabular}

We present several findings. First, we provide a comparison of all the community detection methods on each of the 9 decoy sets along the metrics listed in Section 2. This comparison allows us to identify methods that best capture the organization of the decoy datasets, which we then evaluate further along the metrics described in Section 2 in the context of decoy selection. Finally, we analyze in more detail some of the better performing methods, highlighting their promise and directions for further research.

\subsection{Evaluation of Community Structure from Community Detection Methods}

Even controlling the maximum degree of a vertex can result in dense graphs, which challenge the edge betweenness community detection method. As this method terminates in a little over 2 days for only one of the decoy datasets, it is not included in our evaluation, where we focus on the remaining 6 methods.

Figure 1 relates the comparison along two selected metrics, flake odf and separability, wich represent the overall findings. A better partitioning method achieves lower flake ODF and higher separability. The top three methods according to these related metrics and others (data not shown) are Louvain, Greedy Modularity Maximization, and Label Propagation. In particular, this principled comparison points to Louvain and Greedy Modularity Maximization as the top two community detection methods to identify community structure in nngraph-embedded decoy data.
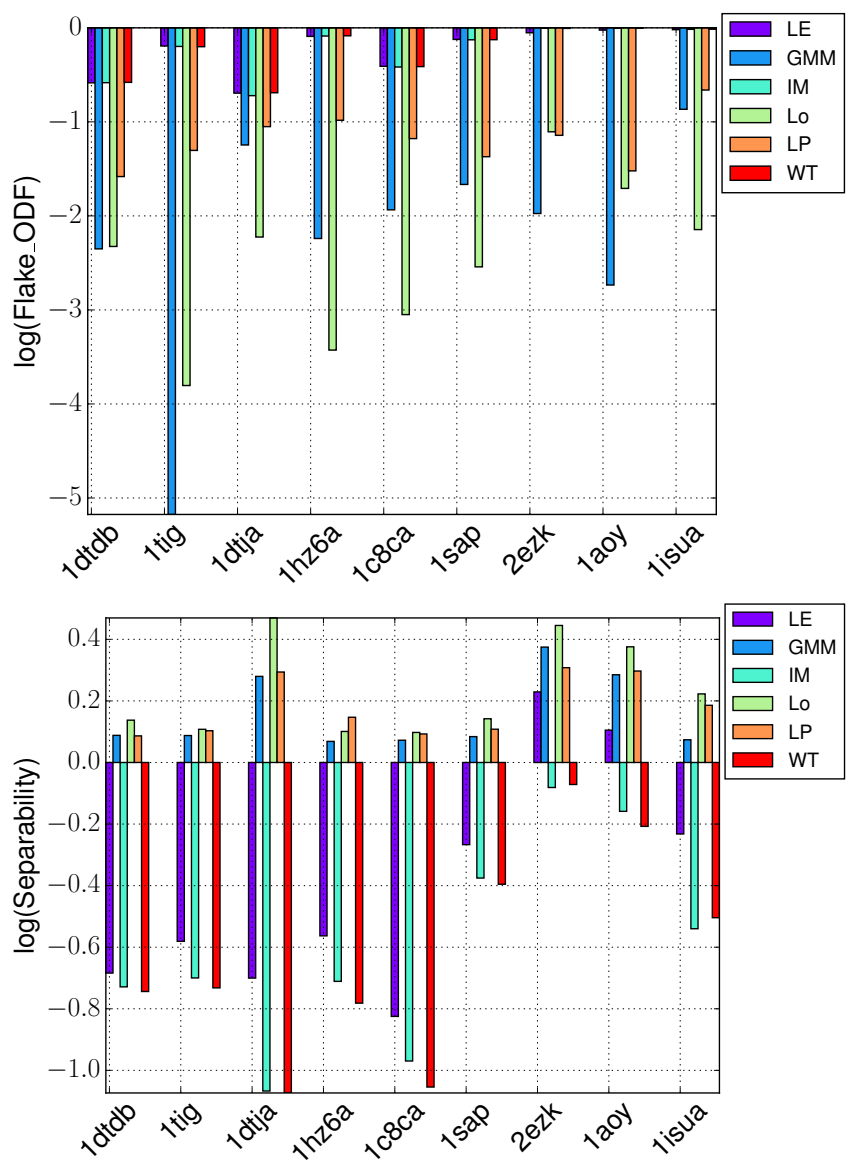

Figure 1: Comparison of 6 community detection methods (encoded by different colors) on each of the 9 test cases along (top panel) flake odf and (bottom panel) separability.

\subsection{Evaluation of Community Selection Strategies for Decoy Selection}

We now focus on evaluating communities selected via the selection strategies described in Section 2 over communities identified on each decoy dataset with the Louvain and the Greedy Modularity Maximization methods. The selected communities are evaluated along the $n$ and $p$ metrics, also described in Section 2. While we have conducted this evaluation for all the 6 community detection methods (data not shown) and on all selection strategies, the quality of the selected communities confirms the superiority of the Louvain and the Greedy Modularity Maximization methods and that of Sel$\mathrm{S}+\mathrm{E}$. This quality is related in Figure 2, which shows $n$ and $p$ values only for $C_{1}$ and $C_{1-3}$ in the interest of space. Results shown are those obtained when using the average energy over decoys in a community to associate an energy with a community, as obtained results are similar when using the minimum energy.

Figure 2 shows that while communities identified via Greedy Modularity Maximization have higher $n$ over communities identified via Louvain, the latter are overall purer. Moreover, the higher values of $p$ are obtained on the easy and medium targets due to the quality of the decoy datasets, where purity reaches even $100 \%$. 

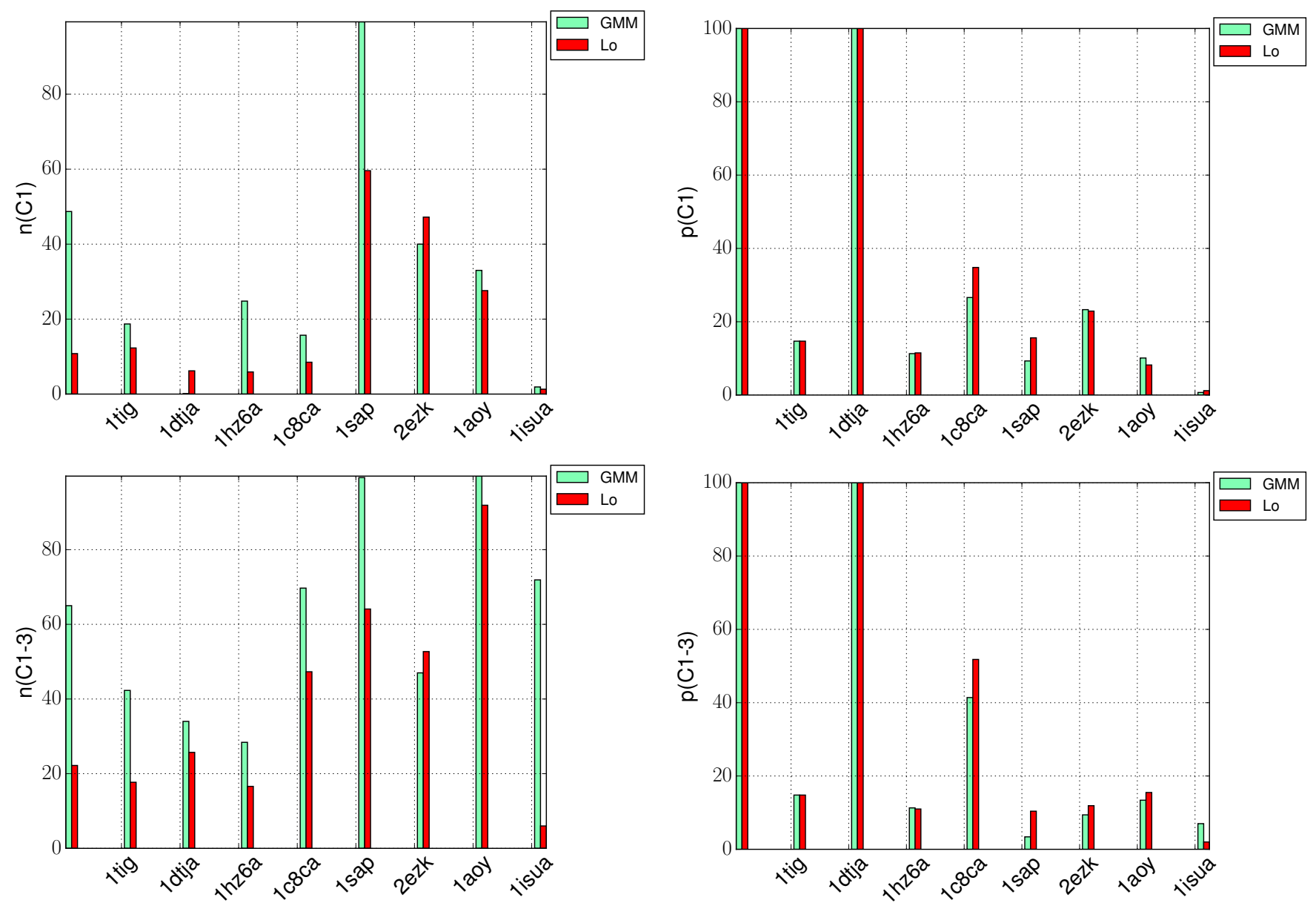

Figure 2: Comparison of communities selected via Sel-S+E on those identified via Louvain and Greedy Modularity Maximization along the $n$ and $p$ metrics. Only metrics on top and top three communities are shown.

These results suggests that a community-based, blind prediction method will offer as prediction only near-native decoys on ensembles of good-quality decoys. The purity of the top communities in the hard cases suffers, most notably due to the low quality of the decoys. Though not shown here in the interest of space, a baseline comparison is conducted with a clustering-based decoy selection that implements leader clustering. On the easy cases, this clusterbased decoy selection does report top clusters with over $98 \%$ purity, but purity quickly decreases over the medium and hard targets, reaching single digits and $0 \%$ (no native decoys detected) on the hard targets. In contrast, the selected communities have appreciable purity even for the hard targets.

To provide further evidence that the Louvain and Greedy Modularity Maximization methods outperform other community detection methods on decoy selection, we show the top three communities selected by the Sel-S+E selection strategy over communities identified by various community detection methods in Figure 3. We limit these results on a medium target (with known native structure under PDB id 1c8ca). Figure 3, which draws decoys by their all-atom energy (Rosetta score12) and their IRMSD from the native structure, color-codes the decoys in the top three communities. The colorcoding allows observing that the structural homogeneity of the top three communities is better when communities are identified with Label Propagation, Louvain, and Greedy Modularity Maximization then Walktrap, and that indeed Louvain and Greedy Modularity Maximization yield the best top communities). Altogether, these results suggest that Louvain and Greedy Modularity Maximization, combined with selection strategies, are promising for advancing research in decoy selection.

\section{CONCLUSION}

The findings show that community detection methods hold promise for organizing structure spaces and warrant further investigation in the context of advancing decoy selection. The work opens further lines of enquiry. The decoys in communities can be further assessed by scoring functions for indicators of nativeness. The quality of the communities themselves can be further improved, by integrating energy in the construction of the decoy nngraph and/or gaging the impact of graph density on the quality of selected communities. Finally, we note that, while the presented work focuses on an application in template-free protein structure prediction, the 

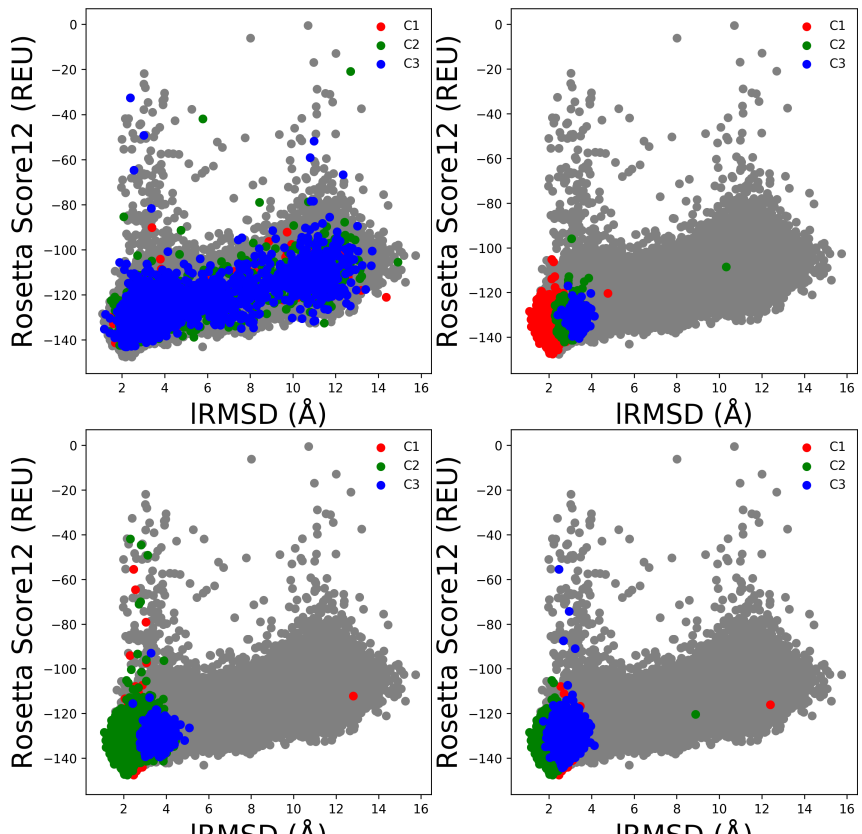

Figure 3: Visualization of color-coded decoys (in terms of energy vs. IRMSD from the native structure) in three top communities selected by the $S+E$ selection strategy over communities identified for a representative medium target (with known native structure under PDB id 1c8ca) via the Walktrap, Label Propagation, Greedy Modularity Maximization, and Louvain methods.

work can be applicable in other settings where an organization of uncomplexed or complexed molecular structure data is needed to reveal states captured in silico.

\section{ACKNOWLEDGMENTS}

This work is supported in part by NSF CCF No. 1421001 and a Jeffress Memorial Trust Award. Computations were run on ARGO, a research computing cluster provided by the Office of Research Computing at George Mason University.

\section{REFERENCES}

[1] N. Akhter and A. Shehu. 2017. From Extraction of Local Structures of Protein Energy Landscapes to Improved Decoy Selection in Template-free Protein Structure Prediction. Molecules 23, 1 (2017), 216.

[2] Arieh Ben-Naim. 1997. Statistical potentials extracted from protein structures are these meaningful potentials? The fournal of Chemical Physics 107, 9 (1997), 3698-3706.

[3] H. M. Berman, K. Henrick, and H. Nakamura. 2003. Announcing the worldwide Protein Data Bank. Nat. Struct. Biol. 10, 12 (2003), 980-980.

[4] Vincent D Blondel, Jean-Loup Guillaume, Renaud Lambiotte, and Etienne Lefeb vre. 2008. Fast unfolding of communities in large networks. Fournal of Statistical Mechanics 2008 (2008), P10008.

[5] D. D. Boehr and P. E. Wright. 2008. How do proteins interact? Science 320, 5882 (2008), 1429-1430

[6] Renzhi Cao, Zheng Wang, Yiheng Wang, and Jianlin Cheng. 2014. SMOQ: a too for predicting the absolute residue-specific quality of a single protein model with support vector machines. BMC bioinformatics 15, 1 (2014), 120.

[7] R. Clausen, B. Ma, R. Nussinov, and A. Shehu. 2015. Mapping the Conformation Space of Wildtype and Mutant H-Ras with a Memetic, Cellular, and Multiscale Evolutionary Algorithm. PLoS Comput Biol 11, 9 (2015), e1004470.
[8] R. Clausen and A. Shehu. 2014. A Multiscale Hybrid Evolutionary Algorithm to Obtain Sample-based Representations of Multi-basin Protein Energy Landscapes. In ACM Conf on Bioinf and Comp Biol (BCB). Newport Beach, CA, 269-278.

[9] Aaron Clauset, M. E. J. Newman, and Cristopher Moore. 2004. Finding community structure in very large networks. Phys. Rev. E 70 (2004), 066111.

[10] M E J Newman. 2006. Finding Community Structure in Networks Using the Eigenvectors of Matrices. Physical review. E 74 (2006), 036104.

[11] Anthony K Felts, Emilio Gallicchio, Anders Wallqvist, and Ronald M Levy. 2002. Distinguishing native conformations of proteins from decoys with an effective free energy estimator based on the opls all-atom force field and the surface generalized Born solvent model. Proteins: Structure, Function, and Bioinformatics 48, 2 (2002), 404-422.

[12] M. Girvan and M. E. J. Newman. 2002. Community structure in social and biological networks. Proc. Natl. Acad. Sci. USA 99, 12 (2002), 7821-7826.

[13] Zhiquan He, Meshari Alazmi, Jingfen Zhang, and Dong Xu. 2013. Protein structural model selection by combining consensus and single scoring methods. PloS one 8, 9 (2013), e74006.

[14] Xiaoyang Jing, Kai Wang, Ruqian Lu, and Qiwen Dong. 2016. Sorting protein decoys by machine-learning-to-rank. Scientific Reports 6 (2016), 31571.

[15] A. Kryshtafovych, A. Barbato, K. Fidelis, B. Monastyrskyy, T. Schwede, and A. Tramontano. 2014. Assessment of the assessment: evaluation of the model quality estimates in CASP10. Proteins 82, Suppl 2 (2014), 112-126.

[16] A. Leaver-Fay and others. 2011. ROSETTA3: an object-oriented software suite for the simulation and design of macromolecules. Methods Enzymol 487 (2011), 545-574.

[17] Tong Liu, Yiheng Wang, Jesse Eickholt, and Zheng Wang. 2016. Benchmarking deep networks for predicting residue-specific quality of individual protein models in CASP11. Scientific reports 6 (2016), 19301.

[18] Stephan Lorenzen and Yang Zhang. 2007. Identification of near-native structures by clustering protein docking conformations. PROTEINS: Structure, Function, and Bioinformatics 68, 1 (2007), 187-194.

[19] Balachandran Manavalan, Juyong Lee, and Jooyoung Lee. 2014. Random forestbased protein model quality assessment (RFMQA) using structural features and potential energy terms. PloS one 9, 9 (2014), e106542.

[20] T. Maximova, D. Carr, E. Plaku, and A. Shehu. 2016. Sample-based Models of Protein Structural Transitions. In ACM Conf Bioinf \& Comp Biol (BCB). Seattle, WA, 128-137.

[21] T. Maximova, R. Moffatt, B. Ma, R. Nussinov, and A. Shehu. 2016. Principles and Overview of Sampling Methods for Modeling Macromolecular Structure and Dynamics. PLoS Comp. Biol. 12, 4 (2016), e1004619.

[22] T. Maximova, ZQi. Zhao, D. B. Carr, E. Plaku, and A. Shehu. 2017. Sample-based Models of Protein Energy Landscapes and Slow Structural Rearrangements. $\mathcal{f}$ Comput Biol 25, 1 (2017), 33-50.

[23] Shokoufeh Mirzaei, Tomer Sidi, Chen Keasar, and Silvia Crivelli. 2016. Purely structural protein scoring functions using support vector machine and ensemble learning. IEEE/ACM transactions on computational biology and bioinformatics (2016).

[24] J. Moult, K. Fidelis, A. Kryshtafovych, T. Schwede, and A. Tramontano. 2014 Critical assessment of methods of protein structure prediction (CASP) - round X. Proteins: Struct. Funct. Bioinf. 82 (2014), 109-115.

[25] Son P Nguyen, Yi Shang, and Dong Xu. 2014. DL-PRO: A novel deep learning method for protein model quality assessment. In Neural Networks (IfCNN), 2014 International foint Conference on. IEEE, 2071-2078.

[26] B. Olson and A. Shehu. 2013. Multi-Objective Stochastic Search for Sampling Local Minima in the Protein Energy Surface. In ACM Conf on Bioinf and Comp Biol (BCB). Washington, D. C., 430-439.

[27] Marcin Pawlowski, Lukasz Kozlowski, and Andrzej Kloczkowski. 2016. MQAPsingle: A quasi single-model approach for estimation of the quality of individual protein structure models. Proteins: Structure, Function, and Bioinformatics 84, 8 (2016), 1021-1028.

[28] Usha Nandini Raghavan, Réka Albert, and Soundar Kumara. 2007. Near linear time algorithm to detect community structures in large-scale networks. Phys. Rev. E 76 (2007), 036106.

[29] M. Rosvall, D. Axelsson, and C. T. Bergstrom. 2009. The map equation. Eur. Phys 7. Special Topics 178 (2009), 13-23.

[30] E. Sapin, D. B. Carr, K. A. De Jong, and A. Shehu. 2016. Computing energy landscape maps and structural excursions of proteins. BMC Genomics 17, Suppl 4 (2016), 456.

[31] Karolis Uziela and Björn Wallner. 2016. ProQ2: estimation of model accuracy implemented in Rosetta. Bioinformatics 32, 9 (2016), 1411-1413.

[32] D. Xu and Y. Zhang. 2012. Ab initio protein structure assembly using continuous structure fragments and optimized knowledge-based force field. Proteins: Struct. Funct. Bioinf. 80, 7 (2012), 1715-1735. DOI : http://dx.doi.org/10.1002/prot.24065

[33] J. Yang and J. Leskovec. 2012. Defining and Evaluating Network Communities based on Ground-truth. In Intl Conf on Data Mining (ICDM). 745-754.

[34] Yang Zhang and Jeffrey Skolnick. 2004. SPICKER: A clustering approach to identify near-native protein folds. Fournal of computational chemistry 25, 6 (2004), 865-871. 